\title{
Inclusion Complexes Oriented in Thermotropic Liquid Crystalline Solvents Studied with Carbon-13 NMR
}

\author{
Malgorzata Marjanska ${ }^{\S *}$, Boyd M. Goodson\# ${ }^{\#}$ Franca Castiglione ${ }^{\S \dagger}$, \\ and Alexander Pines ${ }^{\S \ddagger}$ \\ $\S$ Materials Sciences Division, Lawrence Berkeley National Laboratory, Berkeley, California 94720 \\ and \\ Department of Chemistry, University of California, Berkeley, California 94720-1460 \\ \# Department of Chemistry and Biochemistry, Southern Illinois University, Carbondale, IL 62901
}

\footnotetext{
*Present address: Center for Magnetic Resonance Research, University of Minnesota, 20216 th Street SE, Minneapolis, MN 55455. E-mail: gosia@cmrr.umn.edu

†Present address: Biosearch Italia SpA, Via R. Lapetit 34, 21040 Gerenzano (Varese) Italy. E-mail: FCastiglione@biosearch.it

‡To whom correspondance should be addressed. E-mail: pines@cchem.berkeley.edu, fax: (1) 510-486-5744
} 


\begin{abstract}
The inclusion complex of cryptophane-A and chloroform dissolved in two non-chiral liquid crystalline environments was investigated via ${ }^{13} \mathrm{C} N M R$. Stable solutions of oriented complexes were prepared using aromatic (ZLI 1132) and aliphatic (ZLI 1695) thermotropic nematic liquid crystals as solvents; ordering of the complexes was manifested by the ${ }^{1} \mathrm{H}-{ }^{13} \mathrm{C}$ dipolar splitting of the ${ }^{13} \mathrm{C}$ resonance of labeled chloroform. In both solutions, the dipolar splitting for the bound ligands was substantially larger than that obtained for the free ligands, indicating a significant increase in ligand ordering upon complexation - despite the absence of direct contact with the oriented solvent molecules. Moreover, a differential enhancement in ordering was observed for complexed ligands in ZLI 1695 liquid crystals compared to those in ZLI 1132. Finally, application of heteronuclear decoupling to the ZLI 1695 solution resulted in a reduced linewidth for the bound ${ }^{13} \mathrm{C}$ chloroform resonance, suggesting that a significant component of the observed line broadening may originate from intermolecular couplings between host and guest molecules. These results demonstrate the potential for using restored dipolar couplings to investigate structural and dynamical aspects of inclusion complexes in solution.
\end{abstract}




\section{INTRODUCTION}

A number of synthetic organic molecules have been designed to incorporate small neutral guest species within their engineered cavities, thereby forming inclusion complexes via reversible trapping processes (see for example: Refs. [1-7]). Correspondingly, there has been considerable interest in studying the structural and dynamical aspects of ligand binding in these systems. Moreover, as the behavior of organic inclusion complexes is governed by various enthalpic and entropic contributions of more general importance -including van der Waals interactions, solvent effects, relative orientation and motional freedom of the guest, and structural reorganization of the host - such systems may also provide useful models of ligand binding in larger (e.g., biological) molecules.

One such complex-forming molecule is cryptophane-A. Roughly spherical in shape, cryptophane-A is composed of two bowl-shaped cyclotriveratrylene sub-units connected by three aliphatic $\left[\left(\mathrm{CH}_{2}\right)_{2}\right]$ linker groups, forming an interior cavity (Fig. 1). The complexes between cryptophane-A and various guest species have been studied previously with NMR, including those between cryptophane-A and methane [5], chloroform [5,6], and xenon [7-9]. These complexes form readily in tetrachloroethane and exhibit association constants of 131 $\mathrm{M}^{-1}, 860 \mathrm{M}^{-1}[7]$, and $3900 \mathrm{M}^{-1}[9]$, respectively, at $278 \mathrm{~K}$.

Here the preparation and preliminary magnetic resonance investigation of the inclusion complex of cryptophane- $\mathrm{A}$ and ${ }^{13} \mathrm{CHCl}_{3}$ in two non-chiral thermotropic nematic liquid crystalline environments are reported ${ }^{1}$. In the absence of isotropic rotational diffusion (hindered by the anisotropic environment of the liquid crystals [10]), dipolar couplings within the inclusion complexes were partially restored. In the liquid crystalline solutions, a dramatic, differential increase in the dipolar splitting of the ${ }^{13} \mathrm{C}$ resonance for chloroform bound within cryptophane-A was observed (compared to that of uncomplexed chloroform in the liquid crys-

\footnotetext{
${ }^{1}$ Results presented in part at the $43^{\text {rd }}$ Experimental Nuclear Magnetic Resonance Conference, April 14-19, 2002, Pacific Grove, CA.
} 
talline solvent). These results demonstrate the potential for exploiting re-introduced dipolar couplings to probe the structure and dynamics of ligand binding and complex ordering in such systems.

Recent work by Péchiné et al. [11] has investigated the use of (cyclodextrin-based) chiral hosts oriented in aqueous (non-chiral) liquid crystalline environments to perform enantiomeric discrimination of chiral ligands. In this work, it was demonstrated that differential quadrupolar splittings could be observed for $(R)$ and $(S)$ ligands using ${ }^{2} \mathrm{H}$ NMR spectroscopy; the observed splittings for bound ligands were reduced compared to that of free ligands in the liquid crystalline solutions.

\section{EXPERIMENTAL}

Two liquid crystalline solutions - stable for up to seven days - were prepared, respectively employing the organic thermotropic liquid crystals ZLI 1695 and ZLI 1132 (EM Industries, Inc.) as the solvent. ZLI 1695 and ZLI 1132 exhibit a nematic phase from $13^{\circ} \mathrm{C}$ to $72^{\circ} \mathrm{C}$ and from $-40^{\circ} \mathrm{C}$ to $71^{\circ} \mathrm{C}$, respectively. ZLI 1695 has a negative magnetic susceptability anisotropy, and the liquid crystal directors align uniformly in the plane perpendicular to the applied magnetic field. ZLI 1132 has a positive magnetic susceptability anisotropy, and the liquid crystal directors align uniformly parallel to the field. The dissolution of cryptophaneA (synthesized by the A. Collet group, Ecole Normale Supérieure de Lyon) in the liquid crystalline solvents was mediated using $d_{2}$-1,1,2,2-tetrachloroethane (Aldrich). Up to $7 \mathrm{mg}$ of cryptophane-A were dissolved in $25 \mu \mathrm{L}$ of $d_{2}$-tetrachloroethane $\left(\mathrm{C}_{2} \mathrm{D}_{2} \mathrm{Cl}_{4}\right)$. Larger quantities of cryptophane-A gave liquid crystalline solutions with poorer stability. The cryptophane$\mathrm{A} / \mathrm{C}_{2} \mathrm{D}_{2} \mathrm{Cl}_{4}$ solution was then mixed with $\approx 0.4 \mathrm{~g}$ of the liquid crystals. For different runs, the ${ }^{13} \mathrm{CHCl}_{3}$ was added to the sample either prior to or after the cryptophane- $\mathrm{A} / \mathrm{C}_{2} \mathrm{D}_{2} \mathrm{Cl}_{4}$ solution had been mixed with the liquid crystals.

NMR experiments were performed on a Varian Unity Inova 300 spectrometer equipped with a $5 \mathrm{~mm}$ PFG auto-switchable liquid state probe and on a Chemagnetics Infinity 500 
spectrometer equipped with a $7.5 \mathrm{~mm}$ MAS solid state probe. The ${ }^{13} \mathrm{C}$ spectra were recorded at $75.3 \mathrm{MHz}$ with a simple pulse-acquire sequence with RF field strength of $35.7 \mathrm{kHz}$. The spectra were recorded nonspinning with a recycle delay of $6 \mathrm{~s}$ (no field-frequency lock was employed). For variable temperature study, the sample was allowed to equilibrate at each temperature for $30 \mathrm{~min}$. The ${ }^{13} \mathrm{C}$ spectra obtained under $\mathrm{CW}{ }^{1} \mathrm{H}$ heteronuclear decoupling $(38 \mathrm{kHz})$ were recorded at $125.6 \mathrm{MHz}$; sample spinning and field-frequency lock were not employed. A recycle delay of $20 \mathrm{~s}$ was used to guard against the possiblity of sample heating.

\section{RESULTS AND DISCUSSION}

The inclusion complex of cryptophane-A and chloroform has been studied previously in isotropic solutions [5]. In the present work, it is shown that this complex also forms readily in liquid crystalline environments. Information about the complex is obtained from the observed ${ }^{13} \mathrm{C}$ NMR spectra. Figure 2 shows ${ }^{13} \mathrm{C}$ NMR spectra obtained from two solutions containing ${ }^{13} \mathrm{CHCl}_{3}$, cryptophane- $\mathrm{A}$, and $\mathrm{C}_{2} \mathrm{D}_{2} \mathrm{Cl}_{4}$ dissolved in the liquid crystals ZLI 1695 (Fig. 2(A)) and ZLI 1132 (Fig. 2(B)). Both spectra display two sets of doublets and one triplet. The triplet "impurity" arises from partially restored ${ }^{2} \mathrm{H}-{ }^{13} \mathrm{C}$ (naturally abundant) couplings in $\mathrm{C}_{2} \mathrm{D}_{2} \mathrm{Cl}_{4}$ species aligned in the liquid crystals. $\mathrm{C}_{2} \mathrm{D}_{2} \mathrm{Cl}_{4}$ is too large to fit within the binding cavity of cryptophane-A, preventing complexation. However, the presence of two sets of doublets in each spectrum confirms the existence of two environments for the ${ }^{13} \mathrm{CHCl}_{3}$ species: that of "free" ${ }^{13} \mathrm{CHCl}_{3}$ in the bulk liquid crystals, and that of ${ }^{13} \mathrm{CHCl}_{3}$ "trapped" within the cavity of cryptophane-A.

Generally, the ordering of trapped and free ligands will not be identical. Thus, orientationally dependent magnetic resonance interactions (e.g., chemical shift anisotropy (CSA) and dipolar couplings) can be significantly different for ligands in the two environments. The centers of the doublets do not align, indicating differences in the CSA for free and trapped chloroform of $218 \mathrm{~Hz}(2.91 \mathrm{ppm})$ and $424 \mathrm{~Hz}(5.65 \mathrm{ppm})$ for ZLI 1695 and ZLI 1132 solutions, respectively. The higher-intensity doublets (with splittings of $1184 \mathrm{~Hz}$ and $2970 \mathrm{~Hz}$ 
in Figs. 2(A) and 2(B), respectively) are characteristic of the splittings that were observed for ${ }^{13} \mathrm{CHCl}_{3}$ dissolved in the pure ZLI 1695 and ZLI 1132 liquid crystals (not shown), and were assigned to free chloroform in both solutions. Correspondingly, the smaller, broader doublet in each spectrum was the result of ${ }^{13} \mathrm{CHCl}_{3}$ trapped within the cryptophane-A. The splittings for trapped chloroform $(2915 \mathrm{~Hz}$ and $6307 \mathrm{~Hz}$ for Figs. 2(A) and 2(B), respectively) were significantly enhanced compared to the free chloroform in both solutions. The relative integrals of the peaks of free and trapped chloroform are consistent with complete equilibration of the solutions given the cryptophane-A/chloroform association constant (230 $\mathrm{M}^{-1}$ at $\left.298 \mathrm{~K}[5]\right)$.

The observed splitting for ${ }^{13} \mathrm{CHCl}_{3}$ in an anisotropic environment is a sum of the scalar coupling and the re-introduced dipolar coupling, and is given by

$$
\Delta \nu_{13} \mathrm{CH}=J_{13} \mathrm{CH}+2 D^{13} \mathrm{CH}
$$

where $J_{13} \mathrm{CH}$ is the indirect coupling constant (approximately $+215 \mathrm{~Hz}$ for chloroform) and $D^{13} \mathrm{CH}$ is the strength of the direct dipole-dipole interaction. The amount of restored dipolar coupling depends on the degree of solute ordering in the liquid crystalline environment. The magnitude of the order parameter, $S$, in the direction of the $\mathrm{C}-\mathrm{H}$ vector can be calculated from the observed splitting using the relation [12]:

$$
\left|S_{13 \mathrm{C}-\mathrm{H}}\right|=\frac{4 \pi^{2} r^{3}}{\gamma_{H} \gamma_{13} h} D_{{ }_{13} \mathrm{C}-\mathrm{H}},
$$

where $r$ is the internuclear distance (0.1073 $\mathrm{nm}$ for chloroform), $\gamma_{H}$ and $\gamma_{13} C$ are the gyromagnetic ratios for $\mathrm{H}$ and ${ }^{13} \mathrm{C}$, respectively, and $h$ is Planck's constant.

In the ZLI 1695 solution, the order parameters of ${ }^{13} \mathrm{CHCl}_{3}$ free in the bulk liquid crystalline environment and trapped in cryptophane-A are 0.020 and 0.055 respectively, corresponding to a $\approx 2.8$-fold enhancement of $|S|$ for trapped versus free chloroform. The effect of temperature on $|S|$ was studied for chloroform in the ZLI 1695 solution (Fig. 3), with increasingly enhanced ordering observed for both environments at lower temperatures. In the ZLI 1132 solution, the order parameters of ${ }^{13} \mathrm{CHCl}_{3}$ in bulk liquid crystals and cryptophane- 
A are 0.056 and 0.125 respectively, corresponding to $\mathrm{a} \approx 2.2$-fold enhancement of $|S|$ for trapped versus free chloroform.

While the presence of numerous magnetically-active nuclei in host molecules in stronglyalligned liquid crystalline media would generally yield complex and uninterpretable spectra, information about the complex may be obtained from the (far simpler) ligand resonances. The larger order parameters for chloroform trapped within cryptophane-A suggest a higher degree of spatial ordering and reduced motion compared to free chloroform in the liquid crystals, even though the trapped ligands do not come in direct contact with the anisotropic liquid crystalline environment. Additionally, a differential enhancement was observed for the ordering of the guest in ZLI 1695 compared to that attained in ZLI 1132, indicating differences in the overall ordering of the complex (or, differences in the structure or dynamics of the inclusion complex between these two systems).

The high degree of ordering observed for the trapped chloroform is consistent with the tight fit expected for the ligand within the host's cavity. The van der Waals volume of chloroform is $\approx 72 \AA^{3}[5]$, whereas the corresponding cavity volume for cryptophane-A is $\approx 95$ $\AA^{3}$ when the linker groups are in an extended anti configuration [9]. Moreover, the large stabilizing enthalpy of formation and large destabilizing entropy of formation $(\Delta \mathrm{H}=-34.3 \mathrm{~kJ}$ $\mathrm{mol}^{-1}$ and $\Delta \mathrm{S}_{0}=-67 \mathrm{~J} \mathrm{~mol}^{-1} \mathrm{~K}^{-1}$, respectively [5]) determined previously for this complex suggest a highly rigid, "crystal-like" structure [7]. It has also been reported [7] that x-ray crystallographic studies of the analogous complex between chloroform and cryptophane-A6 (in which ethoxy groups are attached to the benzene rings of the cyclotriveratrylene subunits, instead of methoxy groups) indicate a highly rigid, closely-packed structure, with the 3 -fold axis of chloroform aligned with that of the host. Indeed, recent crystallographic results have confirmed the same structural arrangement for chloroform and cryptophane-A $[13]$.

The effects of decoupling were also investigated in the ZLI 1695 solution. Under ${ }^{1} \mathrm{H}$ broadband heteronuclear decoupling, the doublets collapsed into two single lines (Fig. 4). 
Also, while the linewidth of the free chloroform was essentially unchanged by the decoupling, the linewidth of trapped chloroform was reduced significantly (from $152 \pm 7 \mathrm{~Hz}$ to $90 \pm 4 \mathrm{~Hz}$, as determined from Lorentzian fits of the peaks). It is likely that this line-narrowing was primarily the result of the loss of intermolecular dipolar coupling between host and guest species. The removal of the CSA broadening of the guest, or the reduction of a distribution of order parameter values (caused, for example, by slight variations in the host environment across the sample) may have also contributed to the observed effect.

\section{CONCLUSIONS}

In summary, the preparation of two stable liquid crystalline matrices containing inclusion complexes of chloroform and cryptophane-A has been demonstrated. Clear differences in the orientationally dependent spectral features were observed between free and trapped ligands in both solutions, including significantly larger dipolar splittings for trapped ligandscorresponding to a two-to-three-fold increase in the order parameter for the $\mathrm{C}-\mathrm{H}$ bond of the guest molecule. The larger order parameters for trapped chloroform are consistent with a higher degree of spatial ordering and reduced motion compared to free chloroform in the bulk liquid crystalline solutions. Additionally a differential enhancement was observed for the ordering of chloroform in the ZLI 1695 liquid crystals compared to that obtained in ZLI 1132. Determining the relative importance of the different contributions to the observed effects (e.g., relative orientation and motional freedom of the guest, and the structure, dynamics, and overall ordering of the host) remains an intriguing problem. Future studies will include the investigation of other ligands of different sizes (and more coupled spins) — as well as other hosts with different cage properties - in both thermotropic and (aqueous) lyotropic liquid crystalline environments. Finally, the presence of signficant intermolecular dipolar couplings induced by the liquid crystalline environment would present an alternative route for magnetization transfer between host and guest spins [14], with an efficiency rivaling that of the Overhauser-type methods exploited within inclusion complexes in isotropic solutions 
$[8,15]$.

\section{ACKNOWLEDGEMENTS}

We are very grateful to our colleagues, Dr. Jean-Pierre Dutasta and Dr. Thierry Brotin, formally from the group of the late Andre Collet for providing samples of cryptophane-A and for helpful comments regarding this paper. The authors thank Professors Elliott E. Burnell, Cynthia J. Jameson and Lyndon Emsley for helpful and enlightening discussions, Professor James W. Emsley for referring the authors to the work later published in Ref. [11], and Dr. Josef Granwehr for comments about the paper. Portions of this work were supported by the Director, Office of Science, Office of Basic Energy Sciences, Materials Sciences Division, of the U.S. Department of Energy under Contract NO. DE-AC03-76SF00098. 


\section{REFERENCES}

[1] Cram, D. J.; Tanner, M. E.; Knobler, C. B. J. Am. Chem. Soc. 1991, 113, 7717-7727.

[2] Robbins, T. A.; Knobler, C. B.; Bellew, D. R.; Cram, D. J. J. Am. Chem. Soc. 1994, $116,111-122$.

[3] Branda, N.; Grotzfeld, R. M.; Valdés, C.; Rebek, J. J. J. Am. Chem. Soc. 1995, 117, $85-88$.

[4] Valdés, C.; Spiz, U. P.; Toledo, L. M.; Kubik, S. W.; Rebek, J. J. J. Am. Chem. Soc. 1995, 117, 12733-12745.

[5] Garel, L.; Dutasta, J. P.; Collet, A. Angew. Chem. Int. Ed. Engl. 1993, 32, 1169-1171.

[6] Canceill, J.; Lacombe, L.; Collet, A. J. Chem. Soc., Chem. Commun. 1987, 3, 219-221.

[7] Bartik, K.; Luhmer, M.; Dutasta, J.-P.; Collet, A.; Reisse, J. J. Am. Chem. Soc. 1998, 120, 784-791.

[8] Luhmer, M.; Goodson, B. M.; Song, Y.-Q.; Laws, D. D.; Kaiser, L.; Cyrier, M. C.; Pines, A. J. Am. Chem. Soc. 1999, 121, 3502-3512.

[9] Brotin, T.; Dutasta, J.-P. Eur. J. Org. Chem. 2003, pages 973-984.

[10] Emsley, J. W.; Lindon, J. C. NMR Spectroscopy Using Liquid Crystal Solvents; Pergamon Press: Oxford, 1975.

[11] Péchiné, J.-M.; Meddour, A.; Courtieu, J. Chem. Commun. 2002, 16, 1734-1735.

[12] Frech, C. B.; Fung, B. M.; Schadt, M. Liq. Cryst. 1988, 3, 713-722.

[13] Dutasta, J.-P. personal communication.

[14] Goodson, B. M. Nuclear Magnetic Resonance of Laser-Polarized Noble Gases in Molecules, Materials, and Organisms; PhD thesis: University of California, Berkeley, 1999. 
[15] Song, Y.-Q.; Goodson, B. M.; Taylor, R. E.; Laws, D. D.; Navon, G.; Pines, A. Angew. Chem. Int. Ed. Engl. 1997, 36, 2368-2370. 


\section{FIGURES}

FIG. 1. Chemical structure of cryptophane-A.

FIG. 2. The ${ }^{13} \mathrm{C}$ NMR spectra of ${ }^{13} \mathrm{C}$-labeled chloroform dissolved in cryptophane-A/ $\mathrm{C}_{2} \mathrm{D}_{2} \mathrm{Cl}_{4} /$ ZLI 1695 (A) and in cryptophane- $\mathrm{A} / \mathrm{C}_{2} \mathrm{D}_{2} \mathrm{Cl}_{4} /$ ZLI 1132 (B) solutions. The spectra were recorded with 2048 and 8192 scans, respectively; spectra shown in (A) and (B) were recorded with spectral widths of 15 and $20 \mathrm{kHz}$, respectively, and are shown baseline-corrected with 5 and $50 \mathrm{~Hz}$ line broadening, respectively.

FIG. 3. Temperature dependence of the order parameter for the ${ }^{13} \mathrm{C}-\mathrm{H}$ bond in chloroform dissolved in a cryptophane-A/C $\mathrm{C}_{2} \mathrm{D}_{2} \mathrm{Cl}_{4} /$ ZLI 1695 solution; and $\diamond$ is the order parameter for trapped chloroform, $\square$ is the order parameter for free chloroform, $\triangle$ is the ratio of the order parameters for trapped and free chloroform. The lines are meant to guide the eye.

FIG. 4. The ${ }^{13} \mathrm{C}$ spectrum under $38 \mathrm{kHz} \mathrm{CW}{ }^{1} \mathrm{H}$ heteronuclear decoupling of ${ }^{13} \mathrm{C}$-labeled chloroform dissolved in a cryptophane-A/ $\mathrm{C}_{2} \mathrm{D}_{2} \mathrm{Cl}_{4} /$ ZLI 1695 solution. The $125.6 \mathrm{MHz}$ carbon spectrum was acquired with 2048 scans, and is shown with $5 \mathrm{~Hz}$ line broadening applied during data analysis. 


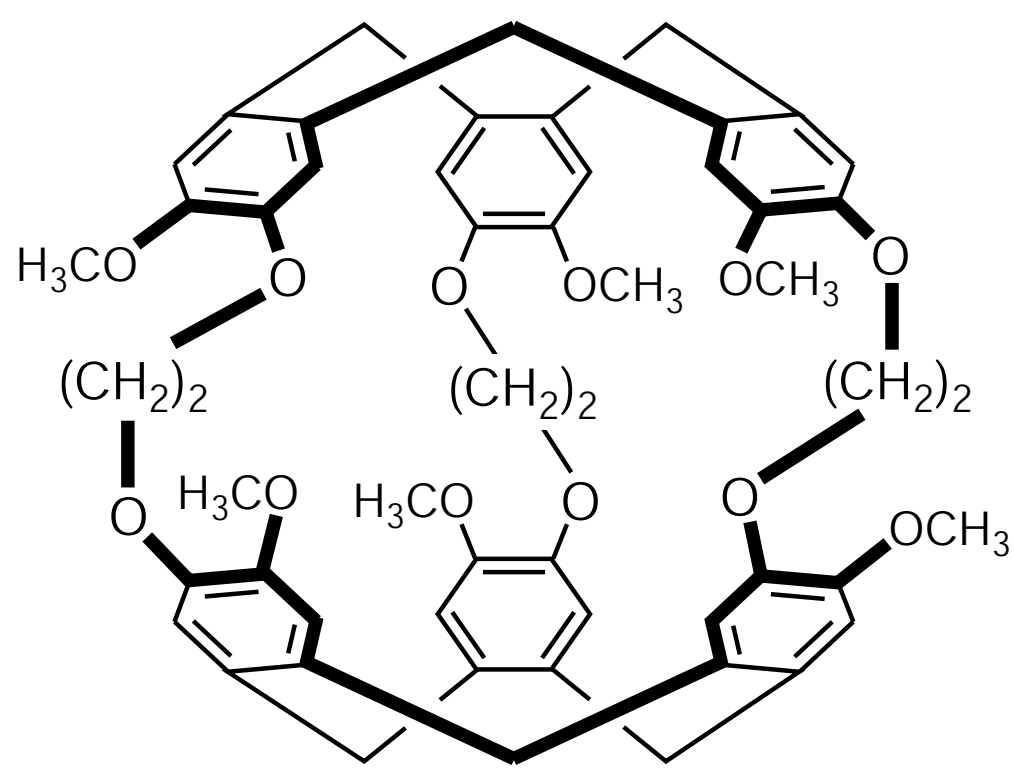


A

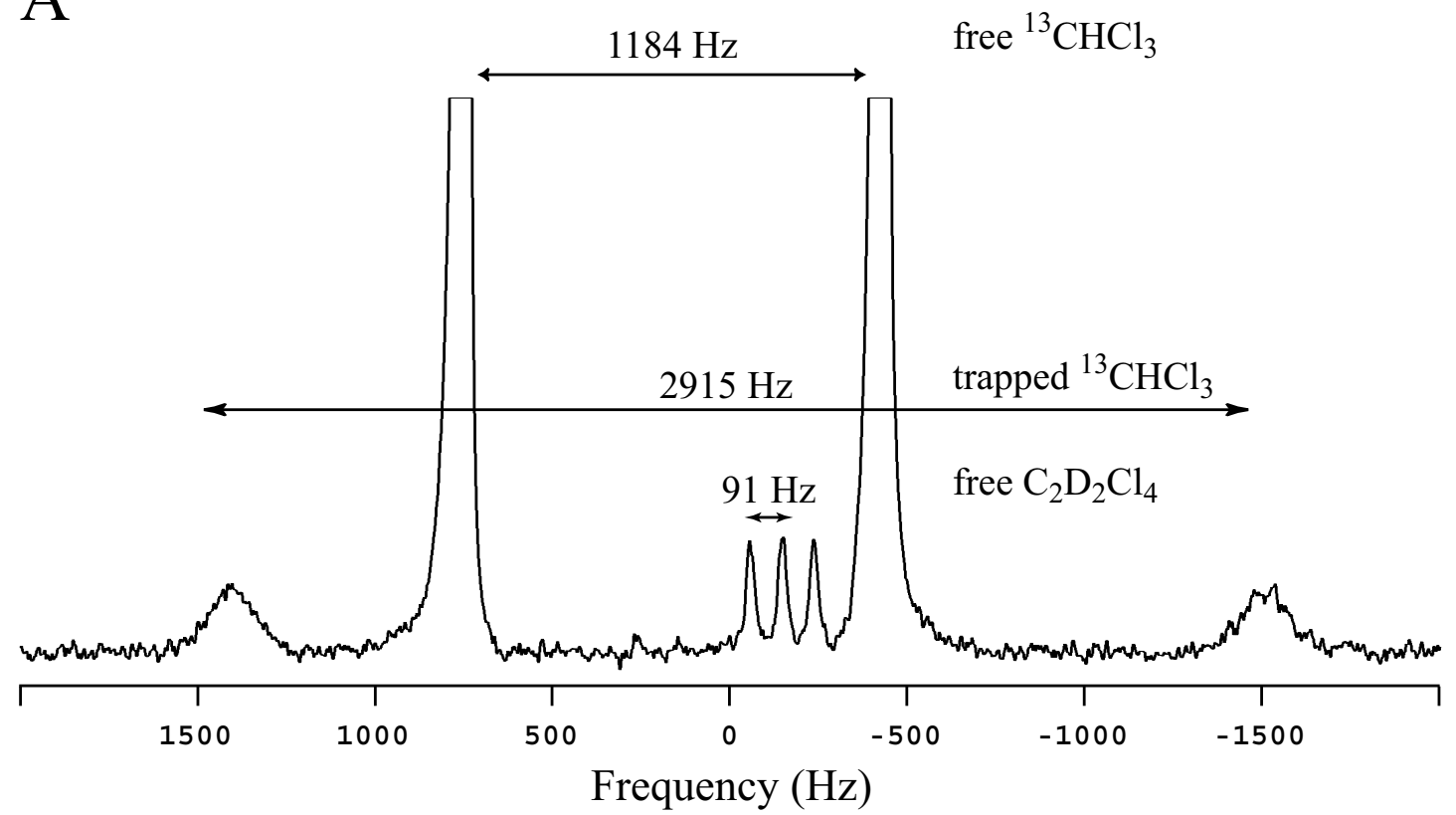

B

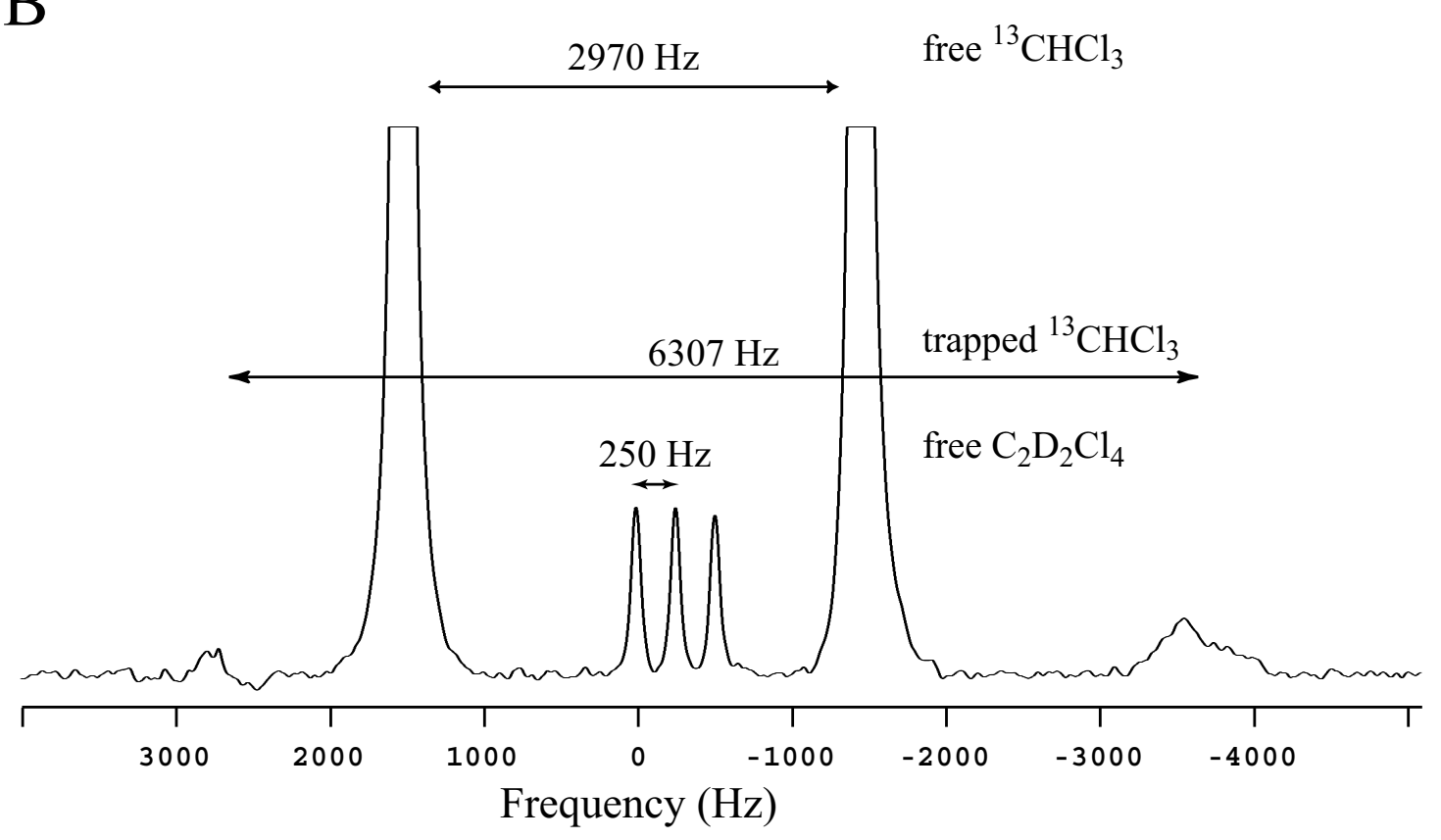




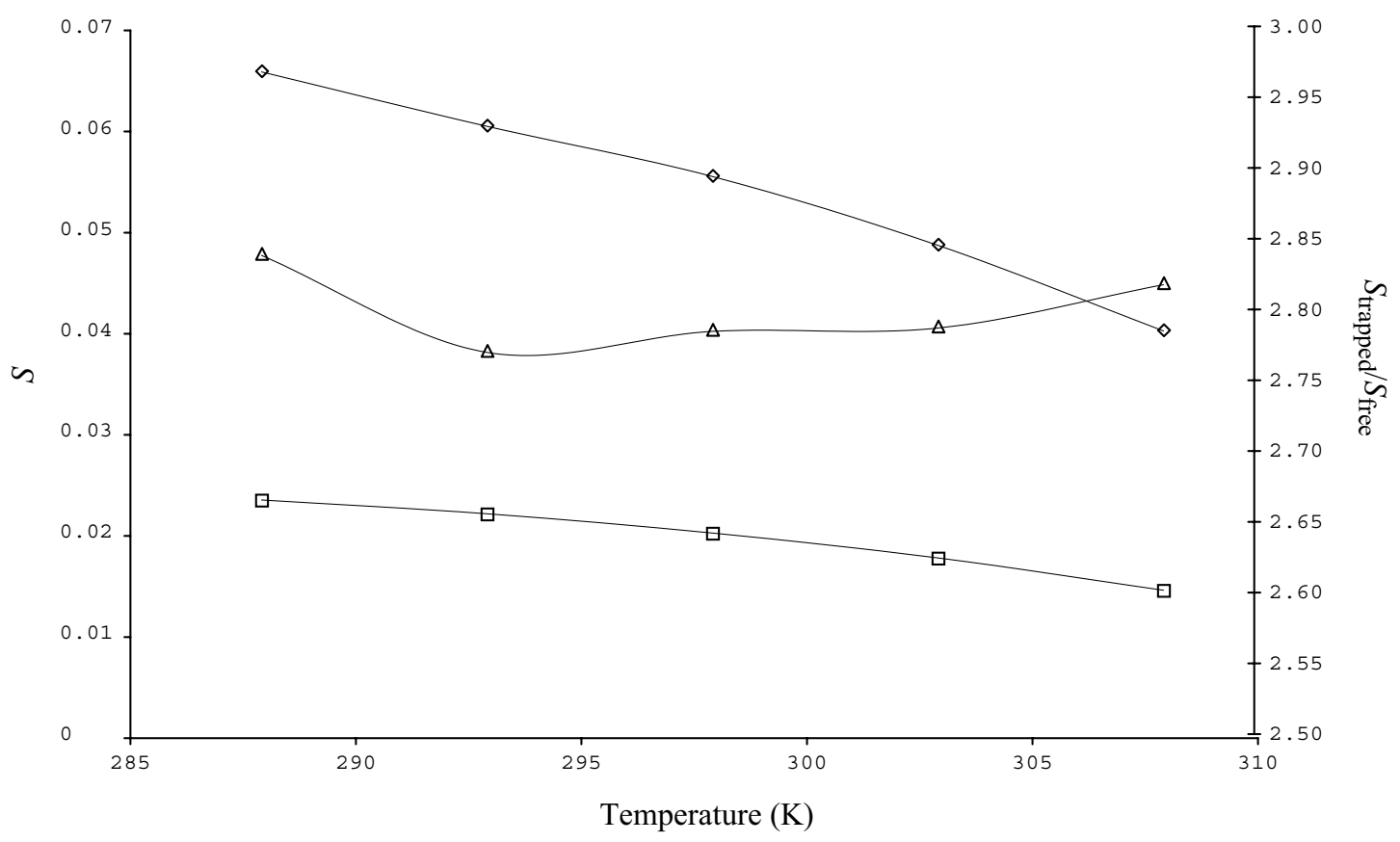




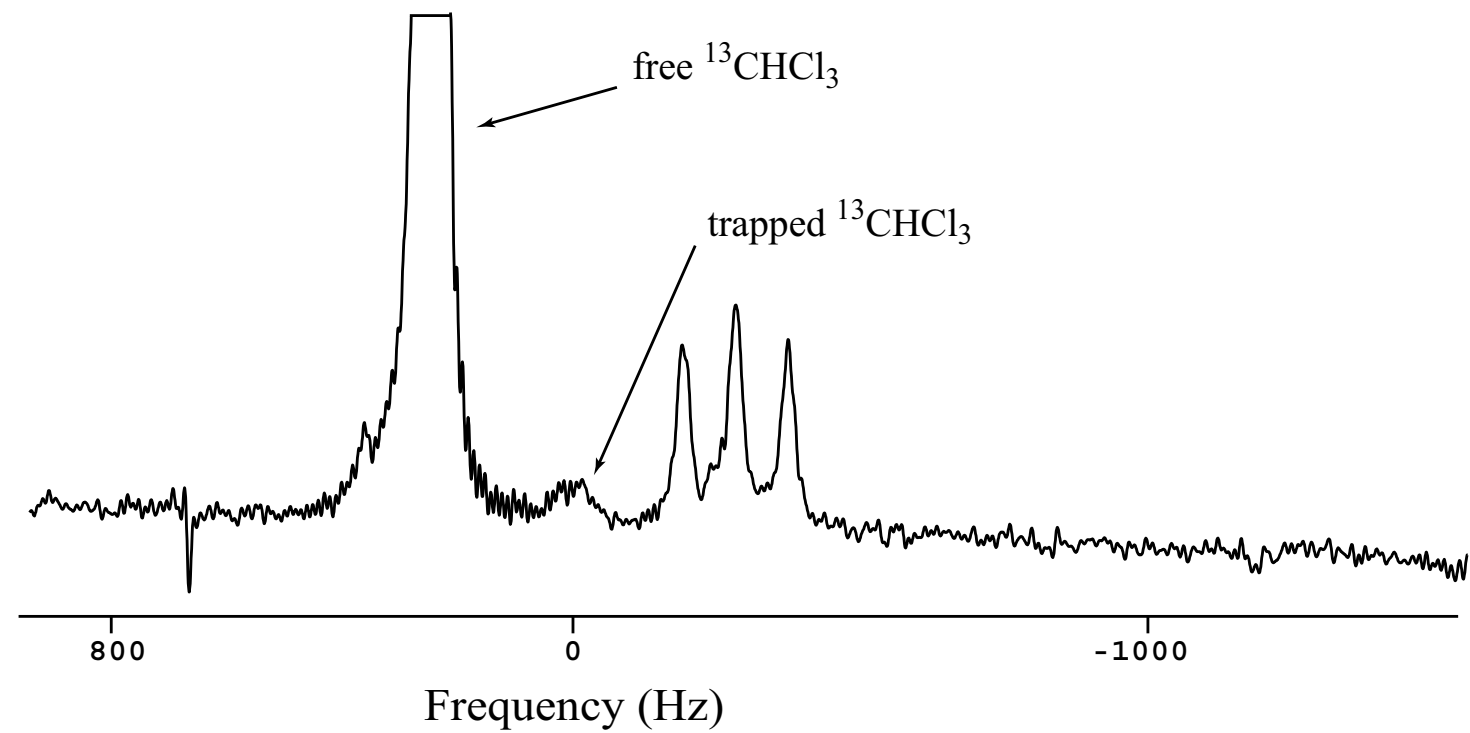

